\title{
Translation of HDAC6 PET Imaging Using [F-18]EKZ-001-cGMP Production and Measurement of HDAC6 Target Occupancy in Nonhuman Primates
}

Sofie Celen ${ }^{1}$, Johanna Rokka ${ }^{2}$, Tonya M Gilbert ${ }^{3}$, Michel Koole ${ }^{4}$, Isabeau Vermeulen ${ }^{1}$, Kim Serdons ${ }^{5}$, Frederick A Schroeder ${ }^{3}$, Florence F Wagner ${ }^{6}$, Tom Bleeser $^{7}$, Baileigh G Hightower ${ }^{2}$ , Jiyun $\mathrm{Hu}^{8}$, Dania Rahal ${ }^{8}, \mathrm{M} \mathrm{Hassan} \mathrm{Beyzavi}^{8}$, Wim Vanduffel ${ }^{2}{ }^{9}$, Koen Van Laere ${ }^{4}{ }^{5}$, Janice $\mathrm{E}$ Kranz $^{3}$, Jacob M Hooker ${ }^{2}$, Guy Bormans ${ }^{1}$, Christopher J Cawthorne ${ }^{4}$

\section{Affiliations}

- $\quad{ }^{1}$ Laboratory for Radiopharmaceutical Research, KU Leuven, Herestraat 49, 3000 Leuven, Belgium.

- $\quad{ }^{2}$ Department of Radiology, Massachusetts General Hospital, Harvard Medical School, Athinoula A. Martinos Center for Biomedical Imaging, Charlestown, Massachusetts 02129, United States.

- $\quad{ }^{3}$ Eikonizo Therapeutics, Inc., Cambridge, Massachusetts 02139, United States.

- $\quad{ }^{4}$ Nuclear Medicine and Molecular Imaging, Department of Imaging and Pathology, KU Leuven, 3000 Leuven, Belgium.

- ${ }^{5}$ Division of Nuclear Medicine, University Hospitals Leuven, Herestraat 49, 3000 Leuven, Belgium.

- ${ }^{6}$ Center for the Development of Therapeutics, Stanley Center for Psychiatric Research, The Broad Institute of MIT and Harvard, Cambridge, Massachusetts 02139, United States.

- $\quad{ }^{7}$ Anesthesiology and Algology, KU Leuven, Herestraat 49, 3000 Leuven, Belgium.

- ${ }^{8}$ Department of Chemistry and Biochemistry, University of Arkansas, Fayetteville, Arkansas 72701 , United States.

- $\quad{ }^{9}$ Laboratory for Neuro- and Psychophysiology, KU Leuven, Herestraat 49, 3000 Leuven, Belgium.

\begin{abstract}
Histone deacetylase 6 (HDAC6) is a multifunctional cytoplasmic enzyme involved in diverse cellular processes such as intracellular transport and protein quality control. Inhibition of HDAC6 can alleviate defects in cell and rodent models of certain diseases, particularly neurodegenerative disorders, including Alzheimer's disease and amyotrophic lateral sclerosis. However, while HDAC6 represents a potentially powerful therapeutic target, development of effective brain-penetrant HDAC6 inhibitors remains challenging. Recently, $\left[{ }^{18} \mathrm{~F}\right]$ EKZ-001 ([ $\left.{ }^{18} \mathrm{~F}\right]$ Bavarostat), a brain-penetrant positron emission tomography (PET) radioligand with high affinity and selectivity toward HDAC6, was developed and evaluated preclinically for its ability to bind HDAC6. Herein, we describe the efficient and robust fully automated current Good Manufacturing Practices (cGMP) compliant production method. $\left[{ }^{18} \mathrm{~F}\right]$ EKZ-001 quantification methods were validated in nonhuman primates (NHP) using full kinetic modeling, and $\left[{ }^{18} \mathrm{~F}\right] \mathrm{EKZ}-001$ PET was applied to compare dose-occupancy relationships between two HDAC6 inhibitors, EKZ-317 and ACY-775. $\left[{ }^{18} \mathrm{~F}\right]$ EKZ-001 is CGMP produced with an average decay-corrected radiochemical yield of $14 \%$ and an average molar activity of $204 \mathrm{GBq} / \mu \mathrm{mol}$. We demonstrate that a two-tissue compartmental model and Logan graphical analysis are appropriate for $\left[{ }^{18} \mathrm{~F}\right]$ EKZ-001 PET quantification in NHP brain. Blocking studies show that the novel compound EKZ-317 achieves higher target occupancy than ACY-775. This work supports the translation of $\left[{ }^{18} \mathrm{~F}\right]$ EKZ-001 PET for first-in-human studies.
\end{abstract}




\section{Introduction}

Acetylation of the $\varepsilon$-amine of lysine is a common post-translational modification that can markedly alter protein functions. Of the many enzymes that modulate acetylation, histone deacetylases (HDACs) are well-known, largely due to the association of HDACs with epigenetic regulation through chromatin remodeling. However, some HDAC family members do not alter chromatin structure or impact gene expression through histone acetylation, including paralog 6 (HDAC6). HDAC6, localized in the cytoplasm, is an attractive target for therapeutic intervention as its effects on protein functions are isolated from genetic material, potentially leading to an improved safety profile. HDAC6 removes acetyl groups from multiple substrates, including tubulin, $(1,2)$ tau, $(3,4)$ and HSP90, $(5,6)$ and thus influences diverse cellular processes such as intracellular transport(7) and protein quality control.(8) In the brain, inhibition of HDAC6 deacetylase activity is reported to increase axonal transport, $(9,10)$ decrease pathological tau hyperphosphorylation, $(3,11,12)$ and decrease stressinduced glucocorticoid receptor signaling.(13)

Small molecule inhibitors of HDAC6 deacetylase activity ameliorated hallmark pathologies and behaviors in models of Alzheimer's disease, $(9,11,12,14)$ frontotemporal dementia,(15) Parkinson's disease, $(16,17)$ amyotrophic lateral sclerosis, $(10,18)$ and major depressive disorder. $(19,20)$ However, HDAC6 inhibitor therapeutics have advanced to the clinic only for peripheral indications. This may in part be due to a lack of highly potent, selective, brainpenetrant HDAC6 inhibitors. Indeed, high doses of HDAC6 inhibitors are required to achieve brain specific functional effects in animal studies, which is not amenable for human translation due to potential off-target liabilities.

To efficiently develop HDAC6 inhibitor therapeutics for central nervous system indications, in vivo measurement of HDAC6 target engagement in the brain is needed to (1) verify whether a therapeutic passes the blood-brain barrier, (2) determine the relationship between therapeutic dose, target occupancy, and downstream mechanistic biomarkers, and (3) understand therapeutic pharmacokinetics and residence time in humans. Positron emission tomography (PET) offers a noninvasive method to quantify target engagement that is translatable to humans, and notably, PET is the only available method for measuring target engagement in the living brain. PET target engagement studies can be incorporated into early therapeutic clinical trials to validate whether a drug candidate binds to the intended target, in the tissue(s) of interest, in a dose-proportional fashion, for the length of time needed to impact relevant biological mechanisms. Importantly, this approach facilitates selection of the appropriate dosing strategy to carry forward in efficacy trials to best test the therapeutic hypothesis.

Recently, several preclinical HDAC6-selective PET radioligands have been reported,(21-23) including the highly brain-penetrant $\left[{ }^{18} \mathrm{~F}\right]$ Bavarostat.(22) Here, we describe an optimized and fully automated radiosynthesis strategy to produce $\left[{ }^{18} \mathrm{~F}\right]$ Bavarostat (hereafter called $\left[{ }^{18} \mathrm{~F}\right] \mathrm{EKZ}$ 001) under current Good Manufacturing Practice (cGMP) conditions using the Trasis All-InOne (AIO) synthesizer and the validation of this radiopharmaceutical for human use. We then apply $\left[{ }^{18} \mathrm{~F}\right]$ EKZ-001 in fully quantitative nonhuman primate (NHP) PET studies to verify in vivo HDAC6 target engagement in the brain and compare dose-occupancy relationships between two potent and paralog selective HDAC6 inhibitors. Together, this work sets the stage for clinical translation of $\left[{ }^{18} \mathrm{~F}\right] \mathrm{EKZ}-001$ PET and may facilitate the development of brain penetrant HDAC6 inhibitor therapeutics.

\section{Results and Discussion}


cGMP Production of [ $\left.{ }^{18} \mathrm{~F}\right]$ EKZ-001 on the Trasis AIO Synthesizer

$\left[{ }^{18} \mathrm{~F}\right]$ EKZ-001 (Figure 1) was synthesized using the synthesis route described by Strebl et al.(22)

(Figure S1) with significant modifications to obtain a cGMP compliant production process for clinical use. $\left[{ }^{18} \mathrm{~F}\right]$ EKZ-001 synthesis was implemented into a commercial, cassette-based automated radiofluorination module, the Trasis AIO synthesizer (Figure S2). The step-by-step description of the $\left[{ }^{18} \mathrm{~F}\right]$ EKZ-001 production method is described in the Supporting Information. The key optimized production features are summarized in Table S1. A more detailed description of the rationale for the implemented changes can be found in the Supporting Information. The ruthenium complex $(\mathrm{CpRu}(\mathrm{COD}) \mathrm{Cl})$ for the radiosynthesis of $\left[{ }^{18} \mathrm{~F}\right] \mathrm{EKZ}-001$ was prepared from our recently published procedure.(24)

\section{Figure 1}

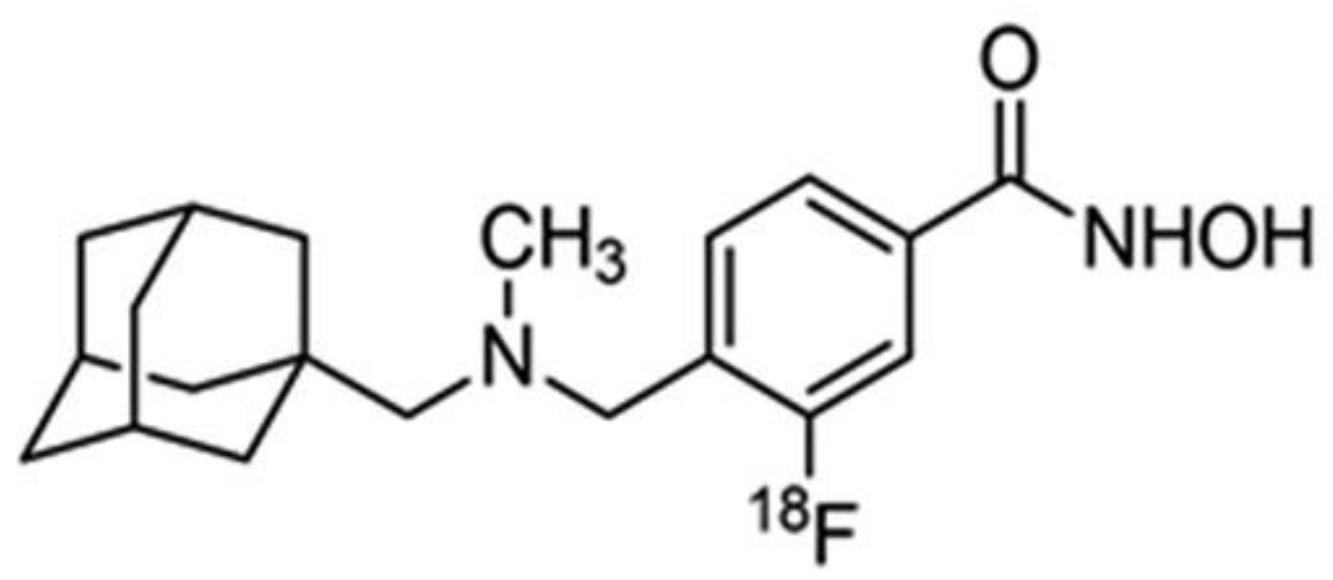

Figure 1. Chemical structure of $\left[{ }^{18} \mathrm{~F}\right]$ EKZ-001 (also known as $\left[{ }^{18} \mathrm{~F}\right]$ Bavarostat).

\section{Quality Control (QC) and Validation for Human Use}

Identity confirmation and determination of the radiochemical purity of the final batch was performed using an analytical radioHPLC system. High signal-to-noise $(\mathrm{S} / \mathrm{N})$ ratios require sharp peaks. Method development was challenging due to the metal ion complex formation properties of the hydroxamate function(25) (see Supporting Information for details on method development). The final optimized HPLC system uses a small dimension $(2 \mathrm{~mm}$ ) metal-free column composed of polyether ether ketone (PEEK) material, heated to $40^{\circ} \mathrm{C}$, with the complexing agent ethylenediaminetetraacetic acid (EDTA) added to the mobile phase and the organic modifier switched from $\mathrm{CH}_{3} \mathrm{CN}$ to EtOH. The limit of detection was $0.11 \mu \mathrm{g} / \mathrm{mL}$ of EKZ-001, which is below the maximum concentration of $0.5 \mu \mathrm{g} / \mathrm{mL}$ that can be injected daily into a human subject.

Three cGMP-compliant productions of $\left[{ }^{18} \mathrm{~F}\right]$ EKZ-001 were performed to validate the production process of the radiotracer for human use. The quality control $(Q C)$ results of the validation runs are summarized in Table 1. A representative analytical QC chromatogram is shown in Figure S5. $\left[{ }^{18} \mathrm{~F}\right]$ EKZ001 was synthesized with high radiochemical purity $>98 \%$ and was identified by coelution with the reference material (retention time difference $<7 \%$ ). 
Table 1. QC Results of the Three Validation Batches of $\left[{ }^{18} \mathrm{~F}\right]$ EKZ-001

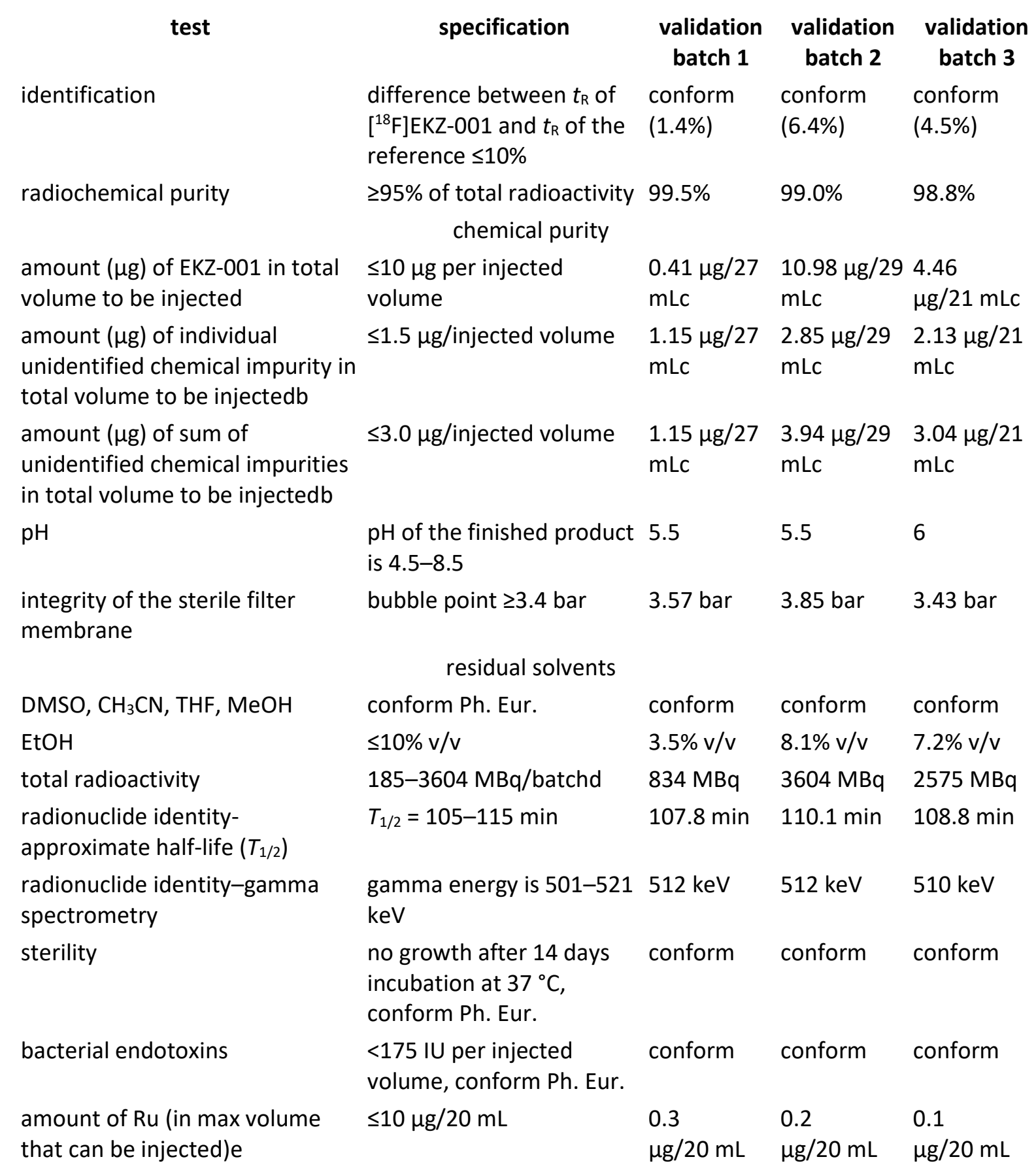


$t_{\mathrm{R}}=$ retention time; $\mathrm{IU}=$ international units; Ph. Eur = European Pharmacopeia; keV = kilo electronvolt; $\mathrm{Ru}=$ ruthenium.

b

Calculated using the UV response factor for EKZ-001.

C

Results are presented for the total batch volume and not the volume to be injected. The volume that is injected is restricted to meet the specification when required.

Based on stability data from validation batches.

e

This test is only performed on the validation batches; more information can be found in the Supporting Information.

When looking at the total batch volume, the chemical impurities and the amount of cold EKZ-001 were slightly higher than the specified maximum. However, due to the high tracer concentration of these batches, only part of the total volume will be injected, and as a result, the amount of chemical impurities and cold EKZ-001 in the injected volume will be below the specified maximum. The bubble point test confirmed the integrity of the sterile filter. The presence of ethanol $(\leq 10 \%)$ prevents radiolysis and minimizes the tracer being retained on the walls of the sterile filter, the vial, and the syringes used to administer the drug product to the subject.(26) All QC results met the specifications. $\left[{ }^{18} \mathrm{~F}\right] \mathrm{EKZ}-001$ was stable (both chemical and radiochemical purities) in its final formulation at room temperature for at least $6 \mathrm{~h}$ post synthesis at a concentration of $126 \mathrm{MBq} / \mathrm{mL}$. The concentration of ruthenium $(\mathrm{Ru})$ in the validation batches was well below the required limit (Table 1 and Supporting Information), and because $\left[{ }^{18} \mathrm{~F}\right] \mathrm{EKZ}-001$ is purified by HPLC, inductively coupled plasma mass spectrometry (ICP-MS) analyses for Ru concentration determination will not be performed on production batches.

Using this validated synthesis method, $\left[{ }^{18} \mathrm{~F}\right]$ EKZ-001 has been synthesized in a total synthesis time of $2 \mathrm{~h}$ with a radiochemical yield of $14 \pm 4 \%$, a radiochemical purity $>98 \%$, and an average molar activity of $204 \pm 175 \mathrm{GBq} / \mu \mathrm{mol}(n=23$ ) for (pre)clinical studies, demonstrating the robustness and reproducibility of our cGMP compliant production method.

\section{Characterization of HDAC6 Inhibitor Small Molecules}

Potency and paralog selectivity were determined for a range of existing and novel candidate HDAC6 inhibitor compounds with an in vitro enzyme inhibition screen using a mobility shift microfluidic technology driven assay. Results, summarized in Table 2, demonstrate high potency and selectivity for ACY-775, EKZ-001, and the novel compound EKZ-317 for HDAC6 versus all other HDAC paralogs. 
These results are supported by HDAC6 target engagement data from in vitro $\left[{ }^{18} \mathrm{~F}\right] \mathrm{EKZ}-001$ autoradiography competition assays using baboon brain tissue.

Table 2. In Vitro Potency, Selectivity, and Target Engagement of HDAC6 Inhibitorsa

\begin{tabular}{|c|c|c|c|c|c|c|c|c|c|c|c|c|c|}
\hline & in vitro & & & & & & & & & & & selectivit & target \\
\hline & $\begin{array}{l}\text { HDAC } \\
\text { inhibitic } \\
n \\
\text { potency } \\
\left({ }^{\prime} C_{50} \text { in }\right. \\
\mu M)\end{array}$ & & & & & & & & & & & $y$ & $\begin{array}{l}\text { engageme } \\
\mathrm{nt}\end{array}$ \\
\hline & 1 & & & & Ila & & 3 & & Ilb & & IV & & \\
\hline $\begin{array}{l}\text { compound } \\
\text { ID }\end{array}$ & 1 & 2 & 3 & 8 & 4 & 5 & 7 & 9 & $\begin{array}{l}\text { HDAC } \\
6\end{array}$ & 10 & 11 & $\begin{array}{l}\text { HDAC6 } \\
\text { vs class } \\
\text { I (fold) }\end{array}$ & $\begin{array}{l}\% \text { blockin } \\
\mathrm{g} \\
\text { efficiency } \\
\text { in tissue }\end{array}$ \\
\hline ACY-738 & 0.07 & $\begin{array}{l}0.2 \\
4\end{array}$ & 0.04 & 1.8 & $\begin{array}{l}\text { N.D } \\
\text {. }\end{array}$ & N.D & $\begin{array}{l}0.0 \\
5\end{array}$ & $\begin{array}{l}\text { N.D } \\
\text {. }\end{array}$ & 0.001 & 0.18 & $\begin{array}{l}\text { N.D } \\
\text {. }\end{array}$ & $40 x$ & $35 \pm 15$ \\
\hline ACY-775 & 2.3 & 7.9 & 1.6 & 3.0 & 3.6 & 2.2 & 3.2 & 11.5 & 0.003 & 2.3 & 8.4 & $533 x$ & $77 \pm 8$ \\
\hline EKZ-001 & 4.7 & $\begin{array}{l}20 . \\
6\end{array}$ & 2.3 & 2.0 & 4.6 & 5.1 & 1.4 & 2.4 & 0.017 & 14.1 & 4.6 & $117 x$ & $100 \pm 10$ \\
\hline EKZ-317 & 47.7 & $\begin{array}{l}36 . \\
5\end{array}$ & 20.8 & $\begin{array}{l}18 . \\
8\end{array}$ & 35.4 & $\begin{array}{l}>10 \\
0\end{array}$ & $\begin{array}{l}38 . \\
7\end{array}$ & $\begin{array}{l}>10 \\
0\end{array}$ & 0.025 & 86.8 & 4.3 & $752 x$ & N.D. \\
\hline $\begin{array}{l}\text { Ricolinost } \\
\text { at }(\mathrm{ACY}- \\
1215)\end{array}$ & 0.16 & $\begin{array}{l}0.4 \\
4\end{array}$ & 0.03 & $\begin{array}{l}0.4 \\
3\end{array}$ & 6.3 & 6.8 & 3.8 & $\begin{array}{l}>10 \\
0\end{array}$ & 0.004 & 0.26 & $\begin{array}{l}>10 \\
0\end{array}$ & $7.5 x$ & $42 \pm 7$ \\
\hline $\begin{array}{l}\text { Tubastatin } \\
\text { A }\end{array}$ & 4.0 & 7.7 & 1.0 & $\begin{array}{l}0.7 \\
0\end{array}$ & 2.3 & 4.2 & $\begin{array}{l}0.8 \\
1\end{array}$ & 2.9 & 0.007 & 9.3 & $\begin{array}{l}>10 \\
0\end{array}$ & $100 x$ & $77 \pm 3$ \\
\hline $\begin{array}{l}\text { Vorinostat } \\
\text { (SAHA) }\end{array}$ & 0.03 & $\begin{array}{l}0.0 \\
8\end{array}$ & $\begin{array}{l}0.00 \\
6\end{array}$ & $\begin{array}{l}0.7 \\
0\end{array}$ & 21.0 & 23.3 & $\begin{array}{l}17 . \\
3\end{array}$ & 23.0 & 0.004 & $\begin{array}{l}0.07 \\
4\end{array}$ & $>30$ & $1.5 x$ & $41 \pm 13$ \\
\hline
\end{tabular}

Compounds were first screened in an in vitro biochemical HDAC activity assay $\left(\mathrm{IC}_{50}\right)$. HDAC6 activity as well as the activity of related HDAC paralogs: HDACs 1, 2, 3, 8 (class I), 4, 5, 7, 9 (class Ila), 10 (class $\mathrm{IIb}$ ), and 11 (class IV) were measured. Compounds were then screened for HDAC6 target engagement in heterologous-blocking in vitro $\left[{ }^{18} \mathrm{~F}\right]$ EKZ-001 autoradiography assays using baboon cerebellum tissue. Blocking efficiency was scaled between $0 \%$ (no inhibitor/baseline condition) and $100 \%$ (selfblock/EKZ-001 condition). Results are the mean measurement of three adjacent tissue sections. N.D. $=$ no data.

\section{Quantitative [18F]EKZ-001 PET Imaging in NHPs}

$\left[{ }^{18} \mathrm{~F}\right]$ EKZ-001 PET was previously performed in rodent and baboon.(22) $\left[{ }^{18} \mathrm{~F}\right]$ EKZ-001 exhibited excellent brain uptake, and self-blocking studies showed high specific binding.(22) Selectivity of $\left[{ }^{18} \mathrm{~F}\right]$ EKZ-001 for HDAC6 was further confirmed in cell-based functional assays and in vitro 
heterologous-blocking autoradiography studies.(22) Here, we further investigate the translational potential of $\left[{ }^{18} \mathrm{~F}\right]$ EKZ-001. Dynamic $\left[{ }^{18} \mathrm{~F}\right]$ EKZ-001 PET studies with arterial blood sampling were performed in macaques ( $n=3$ males) to compare different kinetic modeling approaches. Using the optimal quantitative approach, PET dose-occupancy studies were performed in macaque ( $n=1$ male) to compare HDAC6 target engagement between the novel candidate HDAC6 inhibitor, EKZ-317, and an existing HDAC6 inhibitor tool compound, ACY-775 [19]. Independently, dynamic [ ${ }^{18}$ F]EKZ-001 PET studies with arterial blood sampling were performed in baboon ( $n=1$ female).

The rate of $\left[{ }^{18} \mathrm{~F}\right] \mathrm{EKZ}-001$ metabolism was moderately fast with an intact fraction of approximately $30 \%$ at 30 min postinjection (Figure 2A). Metabolite corrected arterial plasma levels of tracer demonstrated a rapid peak before a sharp decline to approximately $10 \mathrm{~min}$ postinjection, followed by a slower decline until the scan ends (Figure 2B). Average plasma-free fraction, determined in each animal prior to injection of vehicle and tracer, was $4.0 \pm 1.3 \%$.

\section{Figure 2}

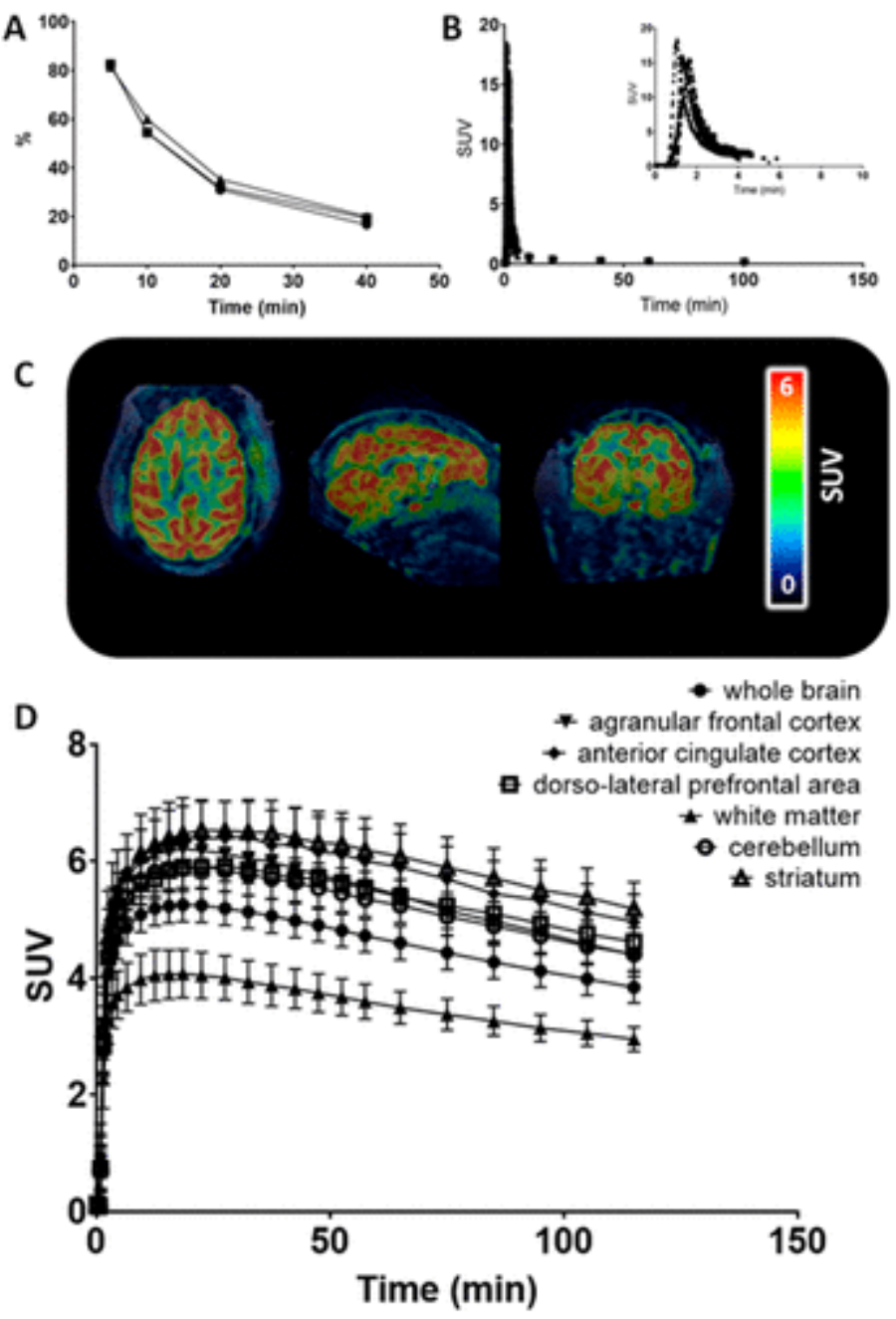

Figure 2. (A) Parent fraction and (B) radiometabolite corrected plasma activity levels of $\left[{ }^{18} \mathrm{~F}\right] E K Z-001$ versus time for macaques $(n=3)$ scanned at baseline (inset $=$ first $10 \mathrm{~min}$ ). (C) Representative 
summed PET SUV images and (D) average regional time activity curves for macaques $(n=3)$ scanned at baseline with $\left[{ }^{18} \mathrm{~F}\right] \mathrm{EKZ}-001$.

As previously reported,(22) uptake of [ ${ }^{18} \mathrm{~F}$ EEKZ-001 peaked at approximately $20 \mathrm{~min}$ in the whole brain and was higher in gray matter compared to white matter. A representative summed standard uptake value (SUV) image (Figure $2 \mathrm{C}$ ) with average SUV time activity curves for selected brain regions (Figure 2D) are shown.

Regional total distribution volume estimates $\left(V_{\mathrm{T}}\right)$ were derived for $\left[{ }^{18} \mathrm{~F}\right] \mathrm{EKZ}-001$ uptake in brain at baseline using both 1-tissue and 2-tissue compartmental models (1TCM and 2TCM), with Akaike information criteria (AIC) to determine the most appropriate kinetic model fit. Data are summarized in Table 3. In general, 2TCM AIC values were lower than for the 1TCM (Figure 3A), therefore demonstrating that the 2TCM is the preferred kinetic model for quantification. Regional $V_{T}$ values were also estimated by a Logan graphical analysis (LGA), using the linear portion of the curve (40 min onward). 2TCM and LGA $V_{\mathrm{T}}$ values were broadly comparable for both baseline and blocking study data, with a highly significant correlation (Table 3 and Figure 3B). Average values were $61 \pm 14$ and 55 \pm 12 (mean \pm standard deviation) for the 2 TCM and LGA, respectively, with the highest uptake observed in amygdala, dorsolateral prefrontal cortex, striatum, and hippocampus and lowest uptake observed in corpus callosum and pons.

Table 3. Distribution Volume Estimates (VT)a Using an Acquisition Time of $120 \mathrm{~min}$ ( $\mathrm{n}=\mathbf{3}$ Macaques)

\section{brain region}

agranular frontal cortex

anterior cingulate cortex

auditory cortex

cerebellum

dorsolateral prefrontal area $71 \pm 26$

orbital prefrontal area

parietal

posterior cingulate cortex

prefrontal area

somatosensory areas

striatum

visual cortex

amygdala

hippocampus

thalamus

pons

corpus callosum
$62 \pm 11$

$49 \pm 9$

$55 \pm 11$

$61 \pm 15$

VT (mL/ccm)

1TCM

$52 \pm 12$

$62 \pm 11$

$52 \pm 13$

$53 \pm 9$

$49 \pm 10$

$69 \pm 15$

$43 \pm 8$

$76 \pm 11$

$60 \pm 12$

$52 \pm 9$

$33 \pm 5$

$34 \pm 5$
AIC

2TCM Logan 1TCM 2TCM

$54 \pm 1451 \pm 12-28 \pm 8 \quad-57 \pm 14$

$73 \pm 7 \quad 59 \pm 11-36 \pm 8 \quad-50 \pm 15$

$54 \pm 1251 \pm 11-28 \pm 7 \quad-40 \pm 10$

$57 \pm 8 \quad 53 \pm 9 \quad-40 \pm 12-54 \pm 14$

$92 \pm 2365 \pm 20-35 \pm 4 \quad-44 \pm 8$

$65 \pm 1159 \pm 10-40 \pm 5-48 \pm 11$

$51 \pm 1050 \pm 11-29 \pm 12-50 \pm 12$

$62 \pm 9 \quad 54 \pm 11-37 \pm 7 \quad-39 \pm 10$

$63 \pm 1558 \pm 13-33 \pm 10-40 \pm 13$

$61 \pm 8 \quad 50 \pm 11-24 \pm 8 \quad-54 \pm 15$

$70 \pm 1564 \pm 12-43 \pm 7 \quad-48 \pm 13$

$47 \pm 7 \quad 43 \pm 8 \quad-11 \pm 6 \quad-8 \pm 5$

$88 \pm 1587 \pm 20-26 \pm 10-41 \pm 4$

$65 \pm 1364 \pm 15-28 \pm 12-49 \pm 17$

$55 \pm 8 \quad 49 \pm 8 \quad-46 \pm 13-43 \pm 17$

$40 \pm 3 \quad 35 \pm 4 \quad-18 \pm 7 \quad-48 \pm 19$

$42 \pm 3 \quad 36 \pm 4-14 \pm 10-24 \pm 15$ 
Derived from 1-tissue and 2-tissue compartmental models (1TCM and 2TCM) and Logan graphical analysis. Akaike information criteria (AIC) values were generated for model fitting to time activity curves of different brain regions.

\section{Figure 3}
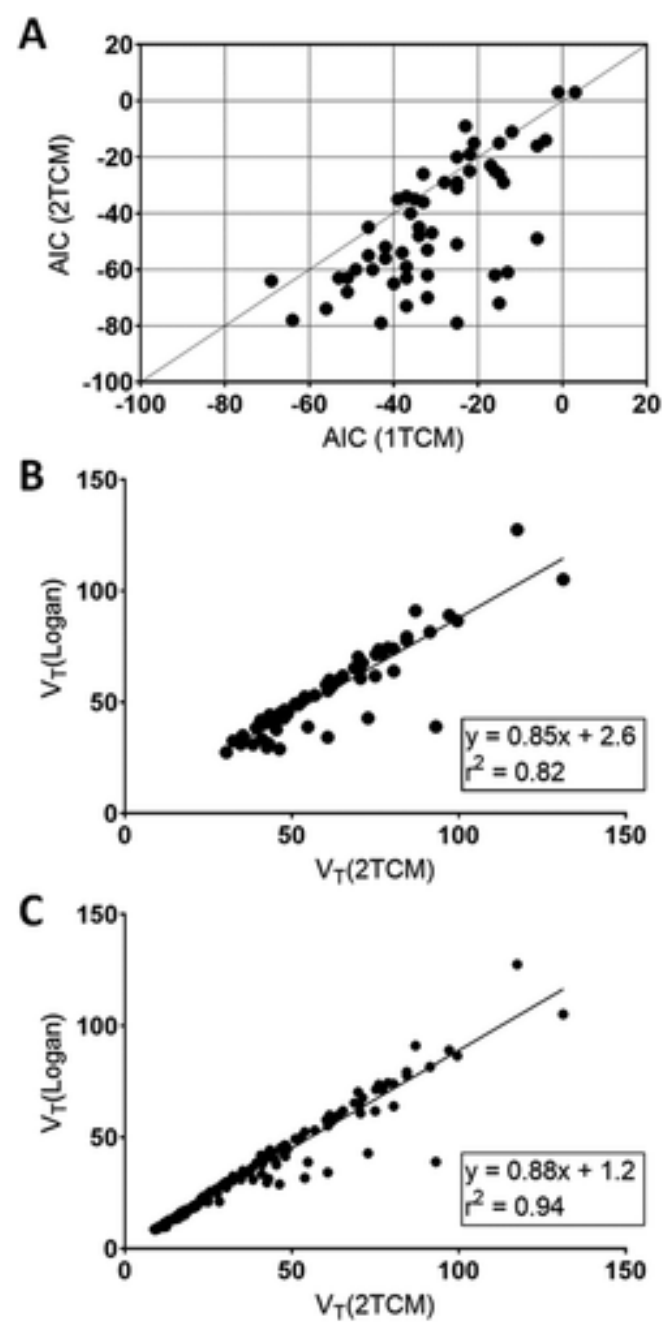

Figure 3. AIC values for 1TCM and 2TCM data fits $(A)$ and correlation of regional distribution volume $\left(V_{\mathrm{T}}\right)$ estimates generated from 2TCM and Logan graphical analysis at baseline (B) ( $n=3$ macaques) and after blocking with EKZ-001 and ACY-775 at 0.1 and $2 \mathrm{mg} / \mathrm{kg}$ (C) ( $n=1$ macaque).

In vivo heterologous-blocking studies were performed with EKZ-317 and ACY-775 at 0.1 and $2 \mathrm{mg} / \mathrm{kg}$ using an intravenous (i.v.) pretreatment paradigm 5 min before $\left[{ }^{18} \mathrm{~F}\right]$ EKZ-001 injection followed by 120 min of dynamic PET scanning (Figure 3C, Figure 4). Target occupancy was estimated via the Lassen plot, where occupancy is given by the slope. Occupancy estimates derived from either LGA (Figure 5A) or 2TCM (Figure 5B) gave comparable results with high correlations. Results indicated high ( $\geq 90 \%$ ) levels of HDAC6 occupancy with EKZ-317 at both doses, whereas only the $2 \mathrm{mg} / \mathrm{kg}$ dose of ACY-775 achieved comparable occupancy. Coupled with the in vitro autoradiography data, this suggests that ACY-775 may have a reduced ability to permeate brain tissue and/or pass the bloodbrain barrier compared to EKZ-317. Nondisplaceable distribution volume $\left(V_{\mathrm{ND}}\right)$ estimates, given by 
the $x$-intercept, are consistent between studies and quantitation methods (approximately 10-15\%), indicating a high degree of specific binding. Blocking with ACY-775 at 0.1 or $2 \mathrm{mg} / \mathrm{kg}$ did not result in increased plasma exposure compared to the same doses of EKZ-317, as indicated by the height of the whole brain and plasma time activity curves (Figure 4 and data not shown), consistent with less peripheral blocking for ACY-775 compared to EKZ-317 and a potential difference in in vivo affinity for HDAC6.

\section{Figure 4}

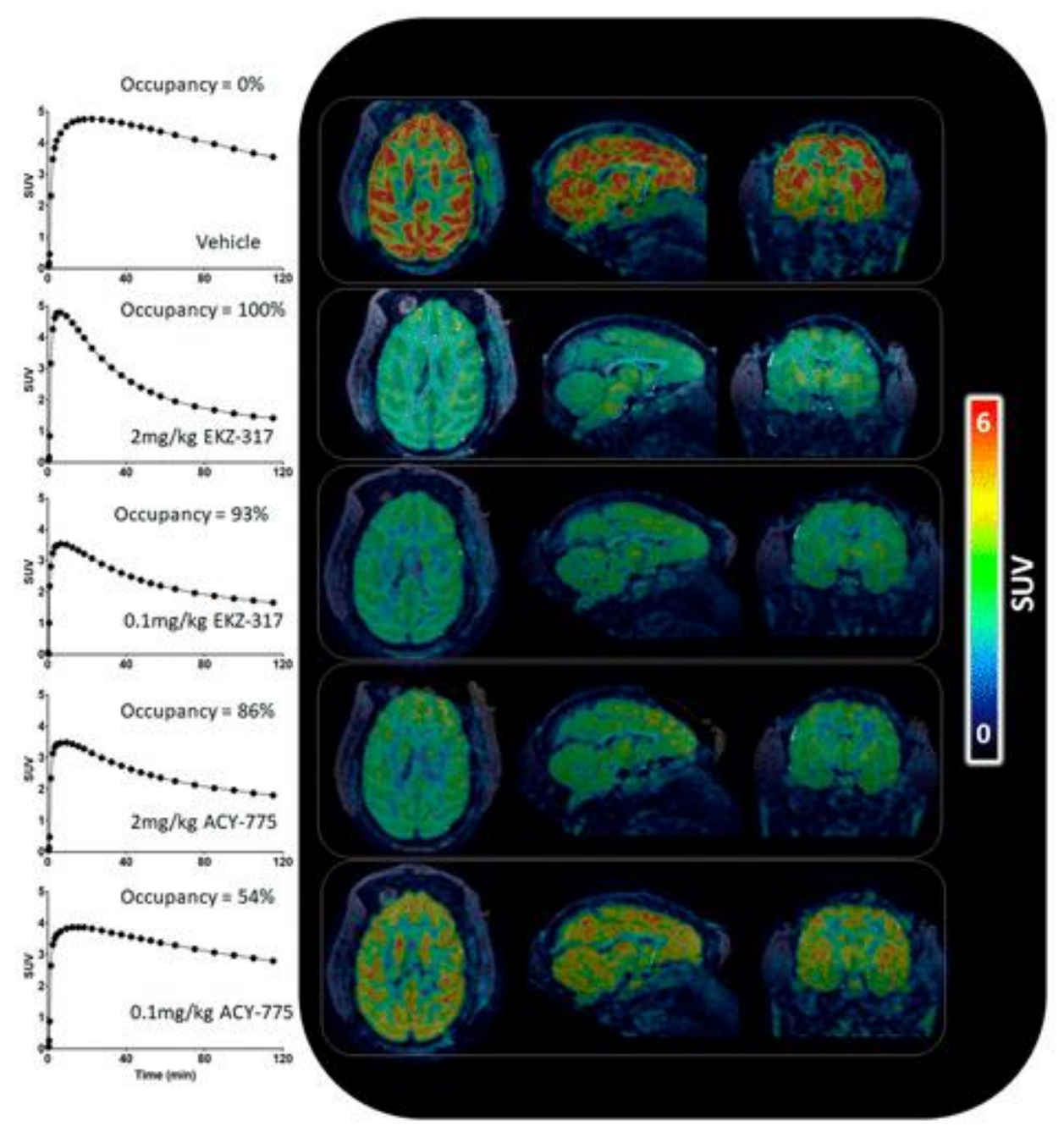

Figure 4. Summed (8-120 min) axial, sagittal, and coronal SUV images of macaque brain (right) with corresponding whole brain time activity curves after pretreatment with vehicle (baseline), EKZ-317, or ACY-775 (left). Compounds were given intravenously at concentrations of 0.1 and $2 \mathrm{mg} / \mathrm{kg} 5 \mathrm{~min}$ before injection of $\left[{ }^{18} \mathrm{~F}\right]$ EKZ-001. Indicated occupancy values were estimated from Logan graphical analysis. 


\section{Figure 5}
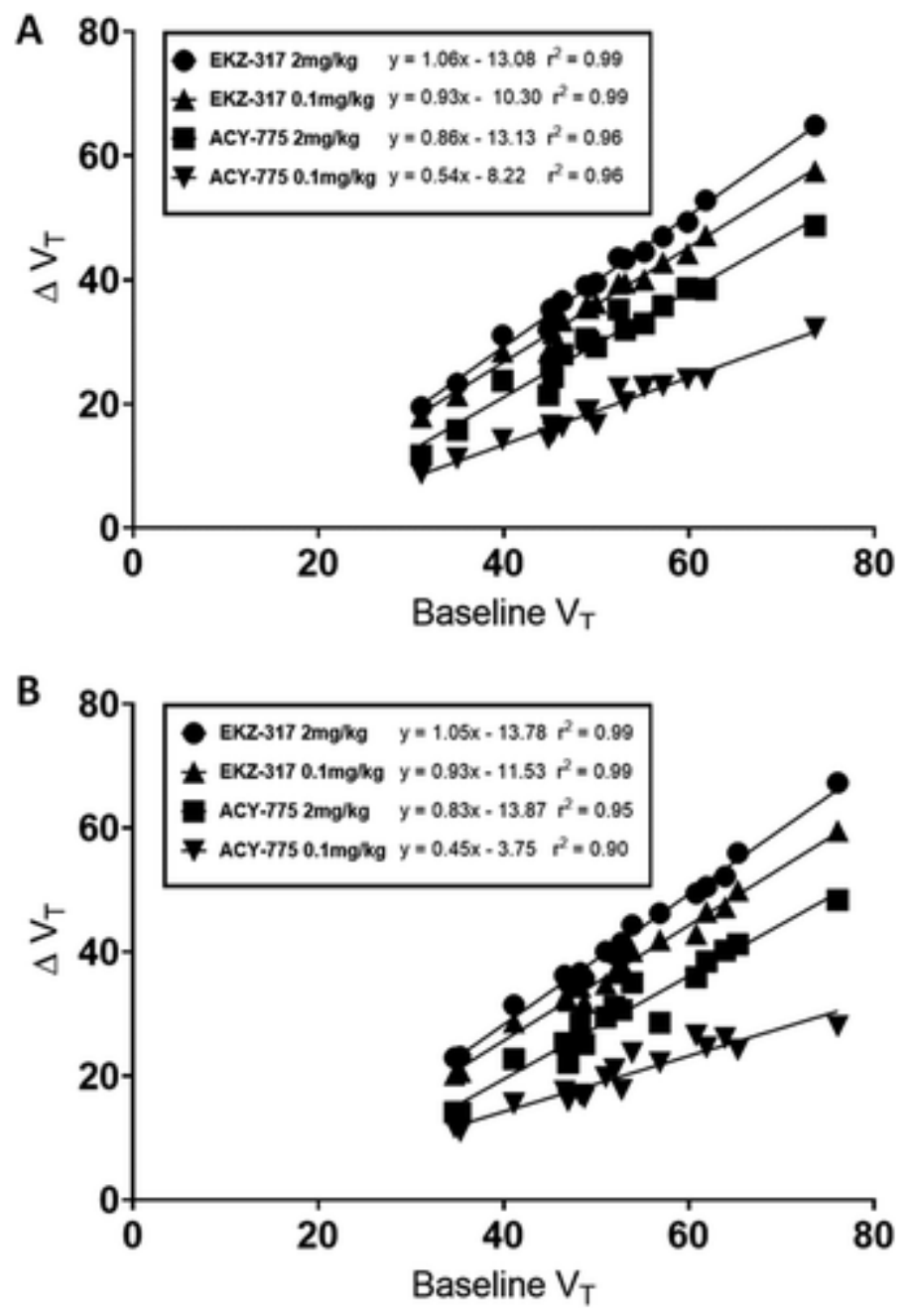

Figure 5. Lassen plots for $\left[{ }^{18} \mathrm{~F}\right]$ EKZ-001 in macaque brain after pretreatment with EKZ-317 and ACY775 using regional distribution volume $\left(V_{T}\right)$ estimates generated from $(A)$ Logan graphical analysis and (B) the $2 \mathrm{TCM}$ ( $n=1$ macaque). Lines are fit by linear regression.

Additional in vivo blocking studies were performed in baboon with the authentic reference compound EKZ-001 at 0.1 and $1 \mathrm{mg} / \mathrm{kg}$, and ACY-775 at $2 \mathrm{mg} / \mathrm{kg}$, using an i.v. pretreatment paradigm 5 min before $\left[{ }^{18} \mathrm{~F}\right]$ EKZ-001 injection and 120 min of dynamic PET scanning (Figure S6A, B). Lassen plots using estimates derived from either LGA (Figure S7A) or 2TCM (Figure S7B) again gave comparable results. Results indicated $\geq 85 \%$ levels of HDAC6 occupancy with EKZ-001 at both doses, whereas ACY-775 at $2 \mathrm{mg} / \mathrm{kg}$ demonstrated markedly lower occupancy, supporting our findings in macaque.

\section{Conclusion}

We developed a fully automated cGMP compliant production method for human use that generates [18F]EKZ-001 with a radiochemical yield of $14 \%$ (decay-corrected) and a radiochemical purity $>98 \%$ in a total synthesis time of $2 \mathrm{~h}$. [18F]EKZ-001 PET showed favorable kinetic properties in NHP brain for calculation of estimates with the 2TCM demonstrating superior fit to the 1TCM. In addition, there was a strong correlation between estimates derived from the 2TCM and LGA, supporting the use of 
graphical analysis. In vivo heterologous-blocking studies indicated that EKZ-317 achieved full HDAC6 occupancy at $2 \mathrm{mg} / \mathrm{kg}$ and greater than $90 \%$ occupancy at $0.1 \mathrm{mg} / \mathrm{kg}$, demonstrating a higher level of target engagement than ACY-775 in brain. This work supports the translation of [18F]EKZ-001 PET for human neuroimaging and target occupancy studies.

\section{Methods}

\section{Radiochemistry}

$\left[{ }^{18} \mathrm{~F}\right]$ EKZ-001 was synthesized using the Trasis AIO synthesizer. A description of materials and equipment is detailed in the Supporting Information. A graphical representation of the cassette designed for the automated synthesis of $\left[{ }^{18} \mathrm{~F}\right]$ EKZ-001 on the Trasis AIO synthesizer is shown in Figure $\mathrm{S} 2$ with each step listed below. Characterization and validation of $\left[{ }^{18} \mathrm{~F}\right]$ EKZ-001 was performed as described in Table S2, which lists the tests, the required batch specifications, and the methods that are used for quality control of the final batch.

\section{In Vitro Potency and Selectivity}

Compounds were screened in a biochemical HDAC activity assay $\left(\mathrm{IC}_{50}\right)$ conducted by Nanosyn (Santa Clara CA, United States). A 12-point concentration curve (in 3-fold serial dilutions starting at 100 or 33.3 $\mu \mathrm{M}$ ) was tested using the microfluidic Caliper LabChip platform, a robust technology for measuring HDAC enzyme activity. Human HDAC6 activity, as well as related human HDAC paralogs: HDACs 1, 2, 3, 8 (class I), 4, 5, 7, 9 (class Ila), 10 (class IIb), and 11 (class IV) were measured.

\section{In Vitro Competition Autoradiography}

Twenty micrometer cryo-sections from male baboon cerebellum tissue were preincubated in $2 \%$ bovine serum albumin (Bioworld, product number 22070008-5)-phosphate buffer solution (1x prepared from 10× PBS, Fisher Scientific, bp399-1) for $30 \mathrm{~min}$. Sections were transferred into a solution containing $37 \mathrm{kBq} / \mathrm{mL}\left[{ }^{18} \mathrm{~F}\right] \mathrm{EKZ}-001$ with or without the blocking agent. Sections were washed in $2 \%$ bovine serum albumin-phosphate buffer solution for $2 \mathrm{~h}$ and then for $2 \mathrm{~min}$ in phosphate buffer solution, dipped in water, and dried. Sections were developed on the imaging plate for $30 \mathrm{~min}$, and the plate was read using the Cyclone (PerkinElmer Inc.). Competitive blocking agents were nonradioactive ACY-738, ACY-775, EKZ-001, Ricolinostat (ACY-1215), Tubastatin A, and Vorinstat (SAHA), all added at $10 \mu \mathrm{M}$. Results were analyzed using Optiquant version 5.0 (PerkinElmer Inc.) and GraphPad Prism (version 5.01) and represent the mean measurement of three adjacent tissue sections per condition.

\section{PET Imaging in Macaques}

Imaging experiments in macaques were conducted according to the Belgian code of practice for the care and use of animals after approval from the local University Ethics Committee for Animals at the Katholieke Universiteit Leuven. Baseline uptake was established in male rhesus monkeys (Macaca mulatta, $n=3,6.5-7.1 \mathrm{~kg}$ ). Occupancy was determined in one animal via PET scanning after pretreatment with 0.1 and $2 \mathrm{mg} / \mathrm{kg}$ of EKZ-317 and ACY-775. All pretreatment studies were performed by i.v. administration at $5 \mathrm{~min}$ prior to $\left[{ }^{18} \mathrm{~F}\right]$ EKZ-001 injection in a volume of $0.4 \mathrm{~mL} / \mathrm{kg}$. Baseline scans used a vehicle containing $5 \% \mathrm{~N}$-methyl-2-pyrrolidone (NMP), 5\% Solutol HS-15, and $90 \% \mathrm{NaCl} 0.9 \%$ for injection. For the blocking scans with EKZ-317 and ACY-775, formulation was performed using 7.5\% NMP, 5\% Solutol HS-15, 30\% PEG-400, and 57.5\% $10 \mathrm{mM}$ citric acid. The macaque was sedated ( $\sim 75 \mathrm{~min}$ before tracer injection) by an intramuscular (i.m.) injection of a combination of $0.3 \mathrm{~mL}$ of Rompun (xylazine $2 \%$ solution) and $0.35 \mathrm{~mL}$ of Nimatek (ketamine 100 $\mathrm{mg} / \mathrm{mL}$ ). About $60 \mathrm{~min}$ after the first injection, the monkey received an additional dose of $0.15 \mathrm{~mL}$ of Rompun and $0.175 \mathrm{~mL}$ of Nimatek via i.v. injection. $\mathrm{O}_{2}$ and $\mathrm{CO}_{2}$ saturation in the blood and heart rate 
were constantly monitored during scanning, and body temperature was maintained via an electronically controlled heating pad.

A venous line was inserted for administration of radiotracer and blocking compounds in one limb. A catheter was placed in the femoral artery in the other limb for arterial blood sampling. Prior to pretreatment/vehicle injection, an arterial blood sample was taken for plasma-free fraction determination.

\section{PET and MR Image Acquisition}

Scans were acquired using the Focus 220 microPET scanner (Concorde Microsystems, Knoxville, TN, United States). Before radiotracer injection, a $10 \mathrm{~min}$ transmission scan using a ${ }^{57} \mathrm{Co}$ source was obtained to assess positioning and for subsequent attenuation correction. A 120 min dynamic PET scan was acquired in list mode concurrently with the injection of $\left[{ }^{18} \mathrm{~F}\right] \mathrm{EKZ}-001$ (185 MBq, manual bolus over $30 \mathrm{~s}$, vena saphena). Data were histogrammed into $4 \times 15 \mathrm{~s}, 4 \times 60 \mathrm{~s}, 5 \times 180 \mathrm{~s}, 8 \times 300 \mathrm{~s}$, and $6 \times 600 \mathrm{~s}$ timeframes and reconstructed using the MAP algorithm (18 iterations, resolution 1.5 $\mathrm{mm}$ ) with attenuation correction into $256 \times 256 \times 95$ pixels. No scatter correction was applied.

A three-dimensional T1-weighted MR scan of each animal was obtained for coregistration purposes on a 3.0 T full-body scanner (Tim Trio Scanner, Siemens) using a magnetization prepared rapid gradient echo (MPRAGE) sequence ( ${ }^{*} \mathrm{tfl} 3 \mathrm{~d} 1$ 1_16) with the following parameters: repetition time 2700 $\mathrm{ms}$, echo time $3.8 \mathrm{~ms}$, inversion time $850 \mathrm{~ms}$, Flip angle $9^{\circ}, 256 \times 208 \times 144$ matrix, $0.6 \mathrm{~mm}$ voxel size.

\section{Arterial Blood Sampling and Plasma Radiometabolite Analysis}

Arterial blood was measured for the first four minutes post $\left[{ }^{18} \mathrm{~F}\right]$ EKZ-001 injection using the Twilite inline blood monitor (Swisstrace, Switzerland). After the initial scan period, the Swisstrace pump was switched off, the arterial blood in the Twilite radiodetector was recovered in EDTA tubes (BD vacutainer, BD, Franklin Lakes, NJ), and arterial blood sampling was continued manually (via a 3-way valve) at preselected time points $(5,10,20$, and 40 min post tracer injection). All collected blood samples were immediately stored on ice to stop metabolism. After centrifugation ( $2330 \mathrm{~g}, 5 \mathrm{~min}$ ), a whole blood sample $(50 \mu \mathrm{L})$ and plasma sample $(50 \mu \mathrm{L})$ were separated and weighed. The remainder of plasma of the six collected samples was processed and analyzed using HPLC to quantify the fraction of intact tracer at the different time points. To about $0.3 \mathrm{~mL}$ of plasma, an equal amount of $\mathrm{CH}_{3} \mathrm{CN}$ was added and the resulting suspension was centrifuged $(2330 \mathrm{~g}, 5 \mathrm{~min}$ ) to separate the precipitated proteins from the supernatant. Next, $0.5 \mathrm{~mL}$ of supernatant was filtered through a syringe filter ( $0.22 \mu \mathrm{m}$; Millipore), diluted with water ( $1 / 2$ of the volume), and spiked with $10 \mu \mathrm{g}$ of authentic EKZ-001. A volume of $0.5 \mathrm{~mL}$ of extract was injected onto an HPLC system consisting of an analytical XTerra column (C18; $5 \mu \mathrm{m}, 4.6 \mathrm{~mm} \times 250 \mathrm{~mm}$, Waters) eluted with a mixture of $0.05 \mathrm{M}$ sodium acetate $(\mathrm{pH} 5.5+0.005 \mathrm{M} \mathrm{EDTA})$ and $\mathrm{CH}_{3} \mathrm{CN}(55: 45 \mathrm{v} / \mathrm{v})$ at a flow rate of $1 \mathrm{~mL} / \mathrm{min}$. For the initial studies, after passing through a radiodetector and UV detector $(280 \mathrm{~nm})$, the HPLC eluate was collected as $1 \mathrm{~mL}$ fractions using an automated fraction collector. Later on, when the radiometabolite profile was known, the HPLC eluate was collected in two fractions (fraction $n^{\circ} 1$ containing the polar radiometabolite(s) and fraction $\mathrm{n}^{\circ} 2$ consisting of the intact tracer). Radioactivity in the filtered plasma (prior to HPLC), filter, and HPLC eluent fractions was all counted in a cross-calibrated welltype gamma counter equipped with a 3-in NaI(TI) well crystal coupled to a multichannel analyzer (Wallac 1480 Wizard, Wallac, Turku, Finland). The results were corrected for background radiation, detector dead-time and physical decay during counting. The dose calibrator, PET camera, gamma counter, and Twilite devices were cross-calibrated with a solution of $\left[{ }^{18} \mathrm{~F}\right] \mathrm{FDG}$ on the day of the experiment. 


\section{Imaging Analysis and Kinetic Modeling}

MRI data from each animal were normalized to a macaque atlas(27) using PFUSIT 4.0 (PMOD, Switzerland). Dynamic PET data were averaged and coregistered to the individual MRI scan before volumes of interest from a publicly available atlas(28) were transformed to PET space to generate time activity curves for kinetic analysis. Kinetic analysis was done in PKIN (PMOD, Switzerland) with the metabolite corrected plasma activity curve was used as an input function for 1- and 2-tissue compartmental models and also for Logan graphical analysis. For the latter, time was set to $40 \mathrm{~min}$ as data were visibly linear at this point.

Drug occupancy levels and were estimated via Lassen plot(29) using $V_{T}$ values derived from the brain regions outlined in Table 3.

The radiotracer-free fraction $\left(f_{\mathrm{p}}\right)$ in plasma was assayed as described previously.(30)

\section{PET Imaging in Baboon}

PET imaging experiments in baboon are detailed in the Supporting Information.

\section{Statistical Analysis}

Statistical analysis was performed in GraphPad Prism (Prism8, Graphpad Inc.) and Microsoft Excel. Pearson correlation analysis was used to determine significance of correlations.

Supporting information: https://pubs.acs.org/doi/10.1021/acschemneuro.0c00074.

\section{References}

This article references 30 other publications.

1.

Hubbert, C., Guardiola, A., Shao, R., Kawaguchi, Y., Ito, A., Nixon, A., Yoshida, M., Wang, X. F., and Yao, T. P. (2002) HDAC6 is a microtubule-associated deacetylase. Nature 417 (6887), 455-8, DOI: $10.1038 / 417455 a$

2.

Haggarty, S. J., Koeller, K. M., Wong, J. C., Grozinger, C. M., and Schreiber, S. L. (2003) Domain-selective small-molecule inhibitor of histone deacetylase 6 (HDAC6)-mediated tubulin deacetylation. Proc. Natl. Acad. Sci. U. S. A. 100 (8), 4389-94, DOI: 10.1073/pnas.0430973100

3.

Cook, C., Carlomagno, Y., Gendron, T. F., Dunmore, J., Scheffel, K., Stetler, C., Davis, M., Dickson, D., Jarpe, M., DeTure, M., and Petrucelli, L. (2014) Acetylation of the KXGS motifs in tau is a critical determinant in modulation of tau aggregation and clearance. Hum. Mol. Genet. 23 (1), 104-16, DOI: 10.1093/hmg/ddt402

4. 
Carlomagno, Y., Chung, D. C., Yue, M., Castanedes-Casey, M., Madden, B. J., Dunmore, J., Tong, J., DeTure, M., Dickson, D. W., Petrucelli, L., and Cook, C. (2017) An acetylationphosphorylation switch that regulates tau aggregation propensity and function. J. Biol. Chem. 292 (37), 15277- 15286, DOI: 10.1074/jbc.M117.794602

5.

Kovacs, J. J., Murphy, P. J., Gaillard, S., Zhao, X., Wu, J. T., Nicchitta, C. V., Yoshida, M., Toft, D. O., Pratt, W. B., and Yao, T. P. (2005) HDAC6 regulates Hsp90 acetylation and chaperonedependent activation of glucocorticoid receptor. Mol. Cell 18 (5), 601- 7, DOI: 10.1016/j.molcel.2005.04.021

6.

Murphy, P. J., Morishima, Y., Kovacs, J. J., Yao, T. P., and Pratt, W. B. (2005) Regulation of the dynamics of hsp90 action on the glucocorticoid receptor by acetylation/deacetylation of the chaperone. J. Biol. Chem. 280 (40), 33792-9, DOI: 10.1074/jbc.M506997200

7.

Dompierre, J. P., Godin, J. D., Charrin, B. C., Cordelieres, F. P., King, S. J., Humbert, S., and Saudou, F. (2007) Histone deacetylase 6 inhibition compensates for the transport deficit in Huntington's disease by increasing tubulin acetylation. J. Neurosci. 27 (13), 3571- 83, DOI: 10.1523/JNEUROSCI.0037-07.2007

8.

Lee, J. Y., Koga, H., Kawaguchi, Y., Tang, W., Wong, E., Gao, Y. S., Pandey, U. B., Kaushik, S., Tresse, E., Lu, J., Taylor, J. P., Cuervo, A. M., and Yao, T. P. (2010) HDAC6 controls autophagosome maturation essential for ubiquitin-selective quality-control autophagy. EMBO J. 29 (5), 969- 80, DOI: 10.1038/emboj.2009.405

9.

Choi, H., Kim, H. J., Kim, J., Kim, S., Yang, J., Lee, W., Park, Y., Hyeon, S. J., Lee, D. S., Ryu, H., Chung, J., and Mook-Jung, I. (2017) Increased acetylation of Peroxiredoxin1 by HDAC6 inhibition leads to recovery of Abeta-induced impaired axonal transport. Mol. Neurodegener. 12 (1), 23, DOI: 10.1186/s13024-017-0164-1

10.

Guo, W., Naujock, M., Fumagalli, L., Vandoorne, T., Baatsen, P., Boon, R., Ordovas, L., Patel, A., Welters, M., Vanwelden, T., Geens, N., Tricot, T., Benoy, V., Steyaert, J., Lefebvre-Omar, C., Boesmans, W., Jarpe, M., Sterneckert, J., Wegner, F., Petri, S., Bohl, D., Vanden Berghe, P., Robberecht, W., Van Damme, P., Verfaillie, C., and Van Den Bosch, L. (2017) HDAC6 inhibition reverses axonal transport defects in motor neurons derived from FUS-ALS patients. Nat. Commun. 8 (1), 861, DOI: 10.1038/s41467-017-00911-y

11. 
Zhang, L., Liu, C., Wu, J., Tao, J. J., Sui, X. L., Yao, Z. G., Xu, Y. F., Huang, L., Zhu, H., Sheng, S. L., and Qin, C. (2014) Tubastatin A/ACY-1215 improves cognition in Alzheimer's disease transgenic mice. J. Alzheimer's Dis. 41 (4), 1193- 205, DOI: 10.3233/JAD-140066

12.

Fan, S. J., Huang, F. I., Liou, J. P., and Yang, C. R. (2018) The novel histone de acetylase 6 inhibitor, MPTOG211, ameliorates tau phosphorylation and cognitive deficits in an Alzheimer's disease model. Cell Death Dis. 9 (6), 655, DOI: 10.1038/s41419-018-0688-5

13.

Espallergues, J., Teegarden, S. L., Veerakumar, A., Boulden, J., Challis, C., Jochems, J., Chan, M., Petersen, T., Deneris, E., Matthias, P., Hahn, C. G., Lucki, I., Beck, S. G., and Berton, O. (2012) HDAC6 regulates glucocorticoid receptor signaling in serotonin pathways with critical impact on stress resilience. J. Neurosci. 32 (13), 4400-16, DOI: 10.1523/JNEUROSCI.563411.2012

14.

Sung, Y. M., Lee, T., Yoon, H., DiBattista, A. M., Song, J. M., Sohn, Y., Moffat, E. I., Turner, R. S., Jung, M., Kim, J., and Hoe, H. S. (2013) Mercaptoacetamide-based class II HDAC inhibitor lowers Abeta levels and improves learning and memory in a mouse model of Alzheimer's disease. Exp. Neurol. 239, 192-201, DOI: 10.1016/j.expneurol.2012.10.005

15.

Selenica, M. L., Benner, L., Housley, S. B., Manchec, B., Lee, D. C., Nash, K. R., Kalin, J., Bergman, J. A., Kozikowski, A., Gordon, M. N., and Morgan, D. (2014) Histone deacetylase 6 inhibition improves memory and reduces total tau levels in a mouse model of tau deposition. Alzheimer's Res. Ther. 6 (1), 12, DOI: 10.1186/alzrt241

16.

Godena, V. K., Brookes-Hocking, N., Moller, A., Shaw, G., Oswald, M., Sancho, R. M., Miller, C. C., Whitworth, A. J., and De Vos, K.J. (2014) Increasing microtubule acetylation rescues axonal transport and locomotor deficits caused by LRRK2 Roc-COR domain mutations. Nat. Commun. 5, 5245, DOI: 10.1038/ncomms6245

17.

Jian, W., Wei, X., Chen, L., Wang, Z., Sun, Y., Zhu, S., Lou, H., Yan, S., Li, X., Zhou, J., and Zhang, B. (2017) Inhibition of HDAC6 increases acetylation of peroxiredoxin1/2 and ameliorates 6-OHDA induced dopaminergic injury. Neurosci. Lett. 658, 114-120, DOI: 10.1016/j.neulet.2017.08.029

18.

Heo, K., Lim, S. M., Nahm, M., Kim, Y. E., Oh, K. W., Park, H. T., Ki, C. S., Kim, S. H., and Lee, S. (2018) A De Novo RAPGEF2 Variant Identified in a Sporadic Amyotrophic Lateral Sclerosis 
Patient Impairs Microtubule Stability and Axonal Mitochondria Distribution. Exp Neurobiol 27 (6), 550-563, DOI: 10.5607/en.2018.27.6.550

19.

Jochems, J., Boulden, J., Lee, B. G., Blendy, J. A., Jarpe, M., Mazitschek, R., Van Duzer, J. H., Jones, S., and Berton, O. (2014) Antidepressant-like properties of novel HDAC6-selective inhibitors with improved brain bioavailability. Neuropsychopharmacology 39 (2), 389400, DOI: $10.1038 / n p p .2013 .207$

20.

Jochems, J., Teegarden, S. L., Chen, Y., Boulden, J., Challis, C., Ben-Dor, G. A., Kim, S. F., and Berton, O. (2015) Enhancement of stress resilience through histone deacetylase 6-mediated regulation of glucocorticoid receptor chaperone dynamics. Biol. Psychiatry 77 (4), 34555, DOI: 10.1016/j.biopsych.2014.07.036

21.

Lu, S., Zhang, Y., Kalin, J. H., Cai, L., Kozikowski, A. P., and Pike, V. W. (2016) Exploration of the labeling of [11C]tubastatin $A$ at the hydroxamic acid site with [11C]carbon monoxide. J. Labelled Compd. Radiopharm. 59 (1), 9-13, DOI: 10.1002/jlcr.3360

22.

Strebl, M. G., Campbell, A. J., Zhao, W. N., Schroeder, F. A., Riley, M. M., Chindavong, P. S., Morin, T. M., Haggarty, S. J., Wagner, F. F., Ritter, T., and Hooker, J. M. (2017) HDAC6 Brain Mapping with [(18)F]Bavarostat Enabled by a Ru-Mediated Deoxyfluorination. ACS Cent. Sci. 3 (9), 1006-1014, DOI: 10.1021/acscentsci.7b00274

23.

Vermeulen, K., Ahamed, M., Luyten, K., and Bormans, G. (2019) Evaluation of [(11)C]KB631 as a PET tracer for in vivo visualisation of HDAC6 in B16.F10 melanoma. Nucl. Med. Biol. 7475,1-11, DOI: 10.1016/j.nucmedbio.2019.05.004

24.

Beyzavi, M. H., Mandal, D., Strebl, M. G., Neumann, C. N., D’Amato, E. M., Chen, J., Hooker, J. M., and Ritter, T. (2017) (18)F-Deoxyfluorination of Phenols via Ru pi-Complexes. ACS Cent. Sci. 3 (9), 944-948, DOI: 10.1021/acscentsci.7b00195

25.

Farkas, E., Enyedy, E. A., and Csoka, H. (2000) Some factors affecting metal ionmonohydroxamate interactions in aqueous solution. J. Inorg. Biochem. 79 (1-4), 20511, DOI: 10.1016/S0162-0134(99)00158-0

26. 
Serdons, K., Verbruggen, A., and Bormans, G. (2008) The presence of ethanol in radiopharmaceutical injections. J. Nucl. Med. 49 (12), 2071, DOI:

10.2967/jnumed.108.057026

27.

McLaren, D. G., Kosmatka, K. J., Oakes, T. R., Kroenke, C. D., Kohama, S. G., Matochik, J. A., Ingram, D. K., and Johnson, S. C. (2009) A population-average MRI-based atlas collection of the rhesus macaque. Neurolmage 45 (1), 52-9, DOI: 10.1016/j.neuroimage.2008.10.058

28.

Seidlitz, J., Sponheim, C., Glen, D., Ye, F. Q., Saleem, K. S., Leopold, D. A., Ungerleider, L., and Messinger, A. (2018) A population MRI brain template and analysis tools for the macaque. Neurolmage 170, 121-131, DOI: 10.1016/j.neuroimage.2017.04.063

29.

Cunningham, V. J., Rabiner, E. A., Slifstein, M., Laruelle, M., and Gunn, R. N. (2010) Measuring drug occupancy in the absence of a reference region: the Lassen plot re-visited. J. Cereb. Blood Flow Metab. 30 (1), 46- 50, DOI: 10.1038/jcbfm.2009.190

30.

Wang, C., Schroeder, F. A., Wey, H. Y., Borra, R., Wagner, F. F., Reis, S., Kim, S. W., Holson, E. B., Haggarty, S. J., and Hooker, J. M. (2014) In vivo imaging of histone deacetylases (HDACs) in the central nervous system and major peripheral organs. J. Med. Chem. 57 (19), 79998009, DOI: $10.1021 /$ jm500872p 